\title{
Analysis of Financial Performance of BUMD Banks in Indonesia
}

\author{
Cahyo Ginarti \\ Politeknik Negeri Medan \\ Jojor Lisbet Sibarani \\ Politeknik Negeri Medan \\ Riswanto \\ Politeknik Negeri Medan
}

\begin{abstract}
This study aims to obtain an explanation and in-depth understanding of the financial performance of regional development banks in Indonesia. Financial performance is measured using Capital Adequacy Ratio (CAR), Non Performing Loans (NPL), Net Interest Margin (NIM), Operating Expenses compared to Operating Income (BOPO), Return on Assets (ROA), and Loan to Deposit Ratio (LDR) ). The specific target of this research is to contribute to the teaching material of financial statement financial analysis, while in the long run the results of this study can be used as a reference for subsequent studies, especially in the field of financial accounting. To achieve this goal, the study was conducted using a quantitative research model with descriptive analysis, of 27 regional development banks registered with OJK. From the analysis it was found that the financial performance of BUMD banks during the observation year still showed an unstable and not as expected movement
\end{abstract}

Keywords : Regional Development Bank, BUMD, Financial Performance.

DOI: $10.7176 / \mathrm{EJBM} / 12-3-19$

Publication date: January $31^{\text {st }} 2020$

\section{Background of Study}

The bank is an intermediary institution in the form of a business entity. As a business entity that functions as an intermediary, banks collect funds from the public in the form of deposits and then distribute them again to the public in the form of credit or other forms "(Khasmir, 2012). In addition, the Bank is also part of the monetary system that has a position as part of economic development.

The Regional Development Bank (BPD) is part of the national banking system. The function and role of the Regional Development Bank (BPD) is very significant. BPD is a bank that is able to move flexibly in the context of regional economic development. This is because the BPD operational network includes services in the regions, and it is not economically possible for private banks to do this.

The Regional Development Bank (BPD), as a regionally owned enterprise (BUMD), is one of the state/public financial institutions (banking). As a banking institution that carries out financial intermediation function, BPD is demanded to increase its role and performance in supporting regional development at any time, especially economic development. But BPD in carrying out business activities must also comply with the principles of transparency and accountability as a public financial institution, including its financial performance. The presence of the BPD is still taken into account in driving the economy, especially in financing development in the regions.

The intense inter-bank competition in the region "forces" each BPD to continue to have a healthy and stable financial performance so that the BPD can become a "leader" in its region because BPD is relatively difficult to expand outside its region or to the national level. However, BPD lending to the non-productive sector has been criticized by the OJK because the quality and quantity have not fully supported regional economic development. BPD financial performance analysis is important to study because it influences how much BPD capacity is in the competition of the national banking industry.

Seeing the magnitude of the role of BPD in encouraging regional development, and the BPD's performance is still not optimal in carrying out its role based on the OJK report (Saragih, 2017), the researcher will conduct research, with the topic 'Analysis of Financial Performance of BUMD Banks in Indonesia'. 


\section{Literature Review}

\section{History of Regional Development Banks in Indonesia}

On May 25, 1960 a Regional Development Bank (Bapindo) was established. The establishment of Bapindo is the beginning of the history of the establishment of the Regional Development Bank in Indonesia. The main task of Bapindo is to assist the government by raising funds from the community in the regions and assisting in financing national development efforts.

Before the establishment of Bapindo, Bank Industri Negara (BIN) was a bank that carried out this function. The function was then transferred to Bapindo in 1960. Besides Bapindo, by issuing Law No. 13/1962 the government also formed the Regional Development Bank (BPD). The establishment of this bank with the main task of helping the government carry out development that is evenly distributed to all regions in Indonesia, by opening operational networks in the regions.

\section{Analysis of Bank Financial Performance}

In analyzing bank financial performance, the approach used is an analysis of bank financial performance or analysis based on CAMEL (capital, assets, management, earnings, and liquidity) associated with the principles of transparency and accountability of public financial management by BPD. CAMEL analysis is based on aspects of how to manage capital / finance of public banks, such as capital adequacy ratio (CAR), return on assets (ROA), analysis of operating expenses vs. operating income (BOPO); rentability analysis, namely the analysis of income or operating income (earnings); and analysis of liquidity (liquidity ratio) including loan to deposit ratio (LDR) and non-performing loan (NPL) credit performance managed by bank management.

\section{Capital Adequacy Ratio (CAR)}

CAR analysis is a ratio that shows the extent to which a bank's ability to anticipate the need for the availability of its own funds for business growth and bear the risk of losses arising in running its business

Formula: $\quad$ CAR $\quad=$ Bank Capital $\times 100 \%$

ATMR

\section{Analysis of Business Efficiency}

Business efficiency analysis is done by calculating "Net Interest Margin (NIM)" and "operating expenses compared to operating income (BOPO)" (Kashmir, 2016)

The BOPO ratio can provide an assessment of the efficiency of banks, including conventional commercial banks and rural banks. If the bank's BOPO ratio in a certain year has decreased from the previous year, the bank's operations will be more efficient. Conversely, if the bank's BOPO ratio in one year has increased from the previous year, the bank's operations will be increasingly inefficient.

Formula:

$$
\text { BOPO }=\frac{\text { Operating Costs } \times 100 \%}{\text { Operating Income }}
$$

\section{Analysis of Profitability and Profitability}

The higher ROA, the greater the rate of return on liquid or productive assets, so that the possibility of banks in problematic conditions is smaller. Profitability / profitability analysis is one method of analyzing bank financial performance to measure the ability of banks to generate profits compared to the capital or assets used by the bank concerned to generate profits. In other words, profitability is the ability of a bank to generate profits for a certain period.

Formula: $\quad \mathrm{ROA}=$ Profit before tax $\mathrm{x} 100 \%$ Average Total Assets

\section{Research Methods}

The stages carried out in this study are as follows 
1. Identifying the phenomena that exist, in this case are issues related to Regional Development Banks, as one of the spearheads of regional development, which is allegedly not yet optimal in carrying out its functions.

2. Conducting a literature review, looking for references regarding indicators used in assessing the financial performance of a bank.

3. Collecting and processing data, in the form of data from the Regional Development Bank's financial statements, 2014 - 2018.

4. Analyze data, discuss and draw conclusions and provide advice.

\section{Research Result}

Calculation of Capital Adequacy Ratio (CAR)

Table 1. Capital Adequacy Ratio (CAR)

\begin{tabular}{|c|c|c|c|c|c|}
\hline \multirow{2}{*}{ BANK } & \multicolumn{5}{|c|}{ CAR ANALYSIS } \\
\hline & 2014 & 2015 & 2016 & 2017 & 2018 \\
\hline Bank Aceh & Increase & Increase & Increase & Increase & Decrease \\
\hline Bank DKI Jakarta & Increase & Increase & Increase & Increase & Decrease \\
\hline Bank Jawa Tengah & Increase & Increase & Increase & Decrease & Decrease \\
\hline Bank Jawa Barat & Increase & Increase & Increase & Increase & Decrease \\
\hline Bank Kalimantan Barat & Increase & Increase & Decrease & Increase & Increase \\
\hline Bank Sumsel Babel & Increase & Increase & Decrease & Increase & Decrease \\
\hline Bank Sumut & Decrease & Increase & Increase & Decrease & Increase \\
\hline Bank Bali & Increase & Increase & Decrease & Menurun & Increase \\
\hline Bank Kaltimtara & Decrease & Increase & Increase & Increase & Decrease \\
\hline Bank Sulawesi Tenggara & Increase & Decrease & Increase & Increase & Increase \\
\hline Bank Sulselbar & Increase & Decrease & Decrease & Decrease & Decrease \\
\hline Bank Sulutgo & Decrease & Decrease & Meningkat & Decrease & Decrease \\
\hline Bank NTB & Increase & Increase & Increase & Decrease & Increase \\
\hline Bank NTT & Increase & Increase & Increase & Decrease & Decrease \\
\hline Bank Papua & Decrease & Increase & Decrease & Increase & Increase \\
\hline Bank Banten & Decrease & Decrease & Increase & Decrease & Decrease \\
\hline Bank Bengkulu & Increase & Increase & Decrease & Increase & Decrease \\
\hline Bank DIY & Increase & Increase & Increase & Decrease & Decrease \\
\hline Bank Jambi & Increase & Decrease & Decrease & Increase & Increase \\
\hline Bank Jawa Timur & Decrease & Decrease & Increase & Increase & Decrease \\
\hline Bank Lampung & Decrease & Increase & Decrease & Increase & Decrease \\
\hline Bank Nagari Sumbar & Increase & Increase & Increase & Increase & Increase \\
\hline Bank Riau Kepri & Decrease & Increase & Decrease & Increase & Decrease \\
\hline Bank Kalimantan Selatan & Increase & Increase & Increase & Decrease & Increase \\
\hline Bank Kalimantan Tengah & Increase & Increase & Decrease & Increase & Decrease \\
\hline $\begin{array}{l}\text { Bank Kalimantan Timur } \\
\text { dan Utara }\end{array}$ & Decrease & Increase & Increase & Increase & Decrease \\
\hline $\begin{array}{l}\text { Improved Financial } \\
\text { Performance }\end{array}$ & 15 & 20 & 16 & 16 & 9 \\
\hline $\begin{array}{l}\text { Declining Financial } \\
\text { Performance }\end{array}$ & 11 & 6 & 10 & 10 & 17 \\
\hline $\begin{array}{l}\text { Fixed Financial } \\
\text { Performance }\end{array}$ & 0 & 0 & 0 & 0 & 0 \\
\hline TOTAL & 26 & 26 & 26 & 26 & 26 \\
\hline $\begin{array}{l}\text { \% Improved Financial } \\
\text { Performance }\end{array}$ & $57.69 \%$ & $76.92 \%$ & $61.54 \%$ & $61.54 \%$ & $34.62 \%$ \\
\hline $\begin{array}{l}\text { \% Declining Financial } \\
\text { Performance }\end{array}$ & $42.31 \%$ & $23.08 \%$ & $38.46 \%$ & $38.46 \%$ & $65.38 \%$ \\
\hline $\begin{array}{l}\% \text { Fixed Financial } \\
\text { Performance }\end{array}$ & $0 \%$ & $0 \%$ & $0 \%$ & $0 \%$ & $0 \%$ \\
\hline$\%$ TOTAL & $100 \%$ & $100 \%$ & $100 \%$ & $100 \%$ & $100 \%$ \\
\hline
\end{tabular}


From the CAR calculation above, it can be seen that the decline in CAR ratio (decline in performance) occurred in 2018 where compared to 2017,17 banks or $65.38 \%$ experienced a decrease in CAR value. The year 2015 showed the best fluctuation, namely only 6 banks or $23.08 \%$ which experienced a decrease in CAR compared to 2014 . In 2016 and 2017 the CAR ratio showed the same performance, namely 10 banks or $38.46 \%$ experienced a decline.

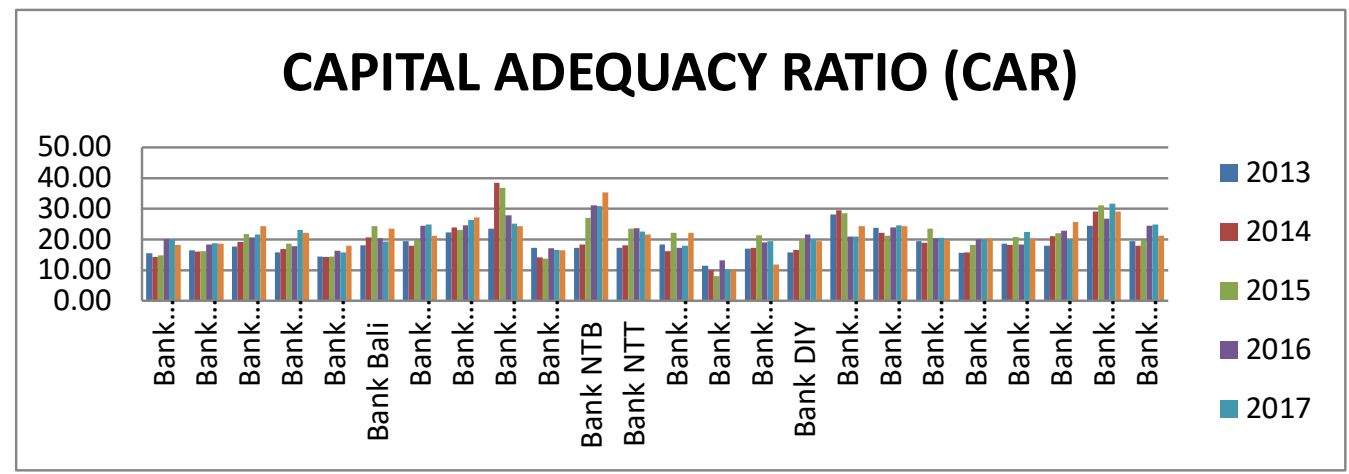

Figure 1. Capital Adequacy Ratio (CAR)

From the bar chart above, it can be seen that Bank Sulselbar and Bank NTB are banks with the highest CAR fluctuations during the observation period. Besides fluctuating with the highest level of fluctuation, the two banks also showed the highest CAR ratio compared to other banks.

\section{Results of NPL Ratio Calculation}

Table 2. Non-Performing Loans (NPL)

\begin{tabular}{|c|c|c|c|c|c|}
\hline \multirow{2}{*}{ BANK } & \multicolumn{5}{|c|}{ ANALYSIS OF NPL } \\
\hline & 2014 & 2015 & 2016 & 2017 & 2018 \\
\hline Bank Aceh & Increase & Increase & Increase & Increase & Increase \\
\hline Bank DKI Jakarta & Decrease & Decrease & Increase & Increase & Increase \\
\hline Bank Jawa Tengah & Decrease & Decrease & Decrease & Decrease & Decrease \\
\hline Bank Jawa Barat & Decrease & Increase & Increase & Increase & Decrease \\
\hline Bank Kalimantan Barat & Decrease & Decrease & Decrease & Decrease & Decrease \\
\hline Bank Sumsel Babel & Increase & Increase & Decrease & Decrease & Increase \\
\hline Bank Sumut & Decrease & Increase & Increase & Increase & Increase \\
\hline Bank Bali & Decrease & Decrease & Increase & Decrease & Decrease \\
\hline Bank Kaltimtara & Increase & Permanent & Permanent & Permanent & Permanent \\
\hline Bank Sulawesi Tenggara & Decrease & Decrease & Increase & Increase & Increase \\
\hline Bank Sulselbar & Increase & Increase & Increase & Decrease & Increase \\
\hline Bank Sulutgo & Decrease & Increase & Increase & Decrease & Decrease \\
\hline Bank NTB & Increase & Increase & Increase & Decrease & Decrease \\
\hline Bank NTT & Decrease & Decrease & Decrease & Decrease & Increase \\
\hline Bank Papua & Decrease & Decrease & Decrease & Increase & Increase \\
\hline Bank Banten & Decrease & Increase & Increase & Increase & Decrease \\
\hline Bank Bengkulu & Decrease & Permanent & Increase & Decrease & Decrease \\
\hline Bank DIY & Decrease & Increase & Decrease & Increase & Decrease \\
\hline Bank Jambi & Decrease & Decrease & Increase & Decrease & Increase \\
\hline Bank Jawa Timur & Increase & Decrease & Decrease & Increase & Increase \\
\hline Bank Lampung & Decrease & Decrease & Decrease & Increase & Decrease \\
\hline Bank Nagari Sumbar & Decrease & Decrease & Decrease & Increase & Increase \\
\hline Bank Riau Kepri & Permanent & Increase & Decrease & Increase & Increase \\
\hline Bank Kalimantan Selatan & Decrease & Decrease & Increase & Decrease & Increase \\
\hline
\end{tabular}




\begin{tabular}{|l|l|l|l|l|l|}
\hline Bank Kalimantan Tengah & Decrease & Increase & Increase & Decrease & Increase \\
\hline $\begin{array}{l}\text { Bank Kalimantan Timur dan } \\
\text { Utara }\end{array}$ & Decrease & Increase & Increase & Increase & Increase \\
\hline Kinerja Keuangan Meningkat & 6 & 12 & 15 & 13 & 15 \\
\hline Kinerja Keuangan Menurun & 19 & 12 & 10 & 12 & 10 \\
\hline Kinerja Keuangan Tetap & 1 & 2 & 1 & 1 & 1 \\
\hline TOTAL & 26 & 26 & 26 & 26 & 26 \\
\hline $\begin{array}{l}\text { \% Improved Financial } \\
\text { Performance }\end{array}$ & $23.08 \%$ & $46.15 \%$ & $57.69 \%$ & $50.00 \%$ & $57.69 \%$ \\
\hline $\begin{array}{l}\text { \% Declining Financial } \\
\text { Performance }\end{array}$ & $73.08 \%$ & $46.15 \%$ & $38.46 \%$ & $46.15 \%$ & $38.46 \%$ \\
\hline $\begin{array}{l}\text { \% Fixed Financial } \\
\text { Performance }\end{array}$ & $3.85 \%$ & $7.69 \%$ & $3.85 \%$ & $3.85 \%$ & $3.85 \%$ \\
\hline$\%$ TOTAL & $100 \%$ & $100 \%$ & $100 \%$ & $100 \%$ & $100 \%$ \\
\hline
\end{tabular}

In contrast to CAR, the NPL ratio shows that the biggest increase (decrease in performance) occurred in 2016 and 2018, as many as 15 banks or $57.69 \%$ experienced an increase in NPL (decreased performance). The best NPL performance actually occurred in 2014, with a decrease in NPL occurred in 19 companies or $73.08 \%$.

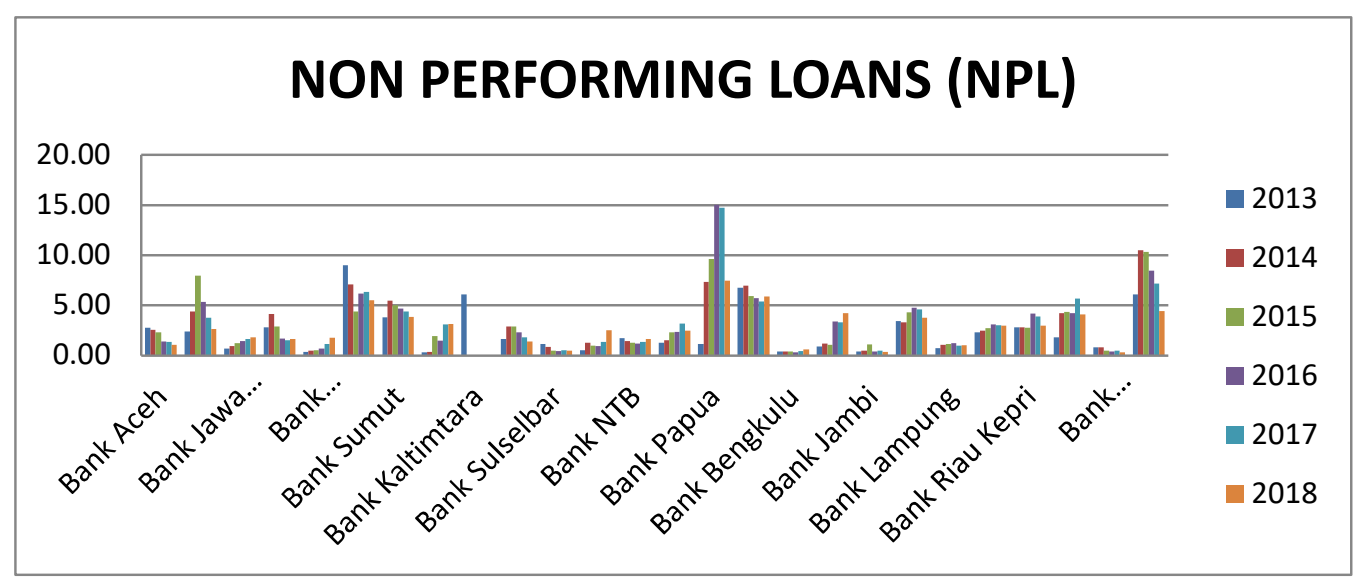

Figure 2. Non Performing Loans (NPL)

From the bar chart above, it can be seen that the Papuan and East Kalimantan Banks are the banks with the highest NPL fluctuations, and both banks also show the highest NPL values compared to other banks.

\section{BOPO Calculation Results}

Table 3. BOPO Calculation

\begin{tabular}{|l|l|l|l|l|l|}
\hline \multirow{2}{*}{ BANK } & \multicolumn{5}{c|}{ BOPO ANALYSIS } \\
\cline { 2 - 6 } & \multicolumn{1}{|c|}{2014} & \multicolumn{1}{c|}{2015} & \multicolumn{1}{c|}{2016} & \multicolumn{1}{c|}{2017} & \multicolumn{1}{c|}{2018} \\
\hline Bank Aceh & Decrease & Decrease & Decrease & Increase & Decrease \\
\hline Bank DKI Jakarta & Decrease & Decrease & Increase & Increase & Increase \\
\hline Bank Jawa Tengah & Decrease & Increase & Decrease & Increase & Increase \\
\hline Bank Jawa Barat & Decrease & Increase & Increase & Decrease & Decrease \\
\hline Bank Kalimantan Barat & Decrease & Decrease & Increase & Increase & Decrease \\
\hline Bank Sumsel Babel & Increase & Increase & Increase & Increase & Increase \\
\hline Bank Sumut & Decrease & Decrease & Increase & Increase & Decrease \\
\hline Bank Bali & Decrease & Decrease & Increase & Decrease & Increase \\
\hline
\end{tabular}




\begin{tabular}{|c|c|c|c|c|c|}
\hline Bank Kaltimtara & Decrease & Decrease & Increase & Increase & Increase \\
\hline Bank Sulawesi Tenggara & Decrease & Decrease & Increase & Decrease & Increase \\
\hline Bank Sulselbar & Increase & Increase & Increase & Decrease & Increase \\
\hline Bank Sulutgo & Decrease & Decrease & Increase & Increase & Decrease \\
\hline Bank NTB & Decrease & Decrease & Decrease & Decrease & Decrease \\
\hline Bank NTT & Decrease & Decrease & Decrease & Increase & Decrease \\
\hline Bank Papua & Decrease & Increase & Decrease & Increase & Increase \\
\hline Bank Banten & Decrease & Decrease & Decrease & Increase & Decrease \\
\hline Bank Bengkulu & Decrease & Decrease & Decrease & Decrease & Decrease \\
\hline Bank DIY & Increase & Increase & Increase & Increase & Decrease \\
\hline Bank Jambi & Decrease & Decrease & Increase & Increase & Decrease \\
\hline Bank Jawa Timur & Decrease & Decrease & Increase & Increase & Decrease \\
\hline Bank Lampung & Increase & Increase & Decrease & Decrease & Decrease \\
\hline Bank Nagari Sumbar & Decrease & Increase & Permanent & Decrease & Increase \\
\hline Bank Riau Kepri & Decrease & Decrease & Increase & Decrease & Decrease \\
\hline Bank Kalimantan Selatan & Increase & Decrease & Decrease & Decrease & Decrease \\
\hline Bank Kalimantan Tengah & Increase & Increase & Decrease & Decrease & Decrease \\
\hline $\begin{array}{l}\text { Bank Kalimantan Timur dan } \\
\text { Utara }\end{array}$ & Decrease & Decrease & Increase & Increase & Increase \\
\hline Improved Financial Performance & 6 & 9 & 15 & 15 & 10 \\
\hline Declining Financial Performance & 20 & 17 & 10 & 11 & 16 \\
\hline Fixed Financial Performance & 0 & 0 & 1 & 0 & 0 \\
\hline TOTAL & 26 & 26 & 26 & 26 & 26 \\
\hline $\begin{array}{l}\text { \% Improved Financial } \\
\text { Performance }\end{array}$ & $23.08 \%$ & $34.62 \%$ & $57.69 \%$ & $57.69 \%$ & $38.46 \%$ \\
\hline $\begin{array}{l}\% \text { Declining Financial } \\
\text { Performance }\end{array}$ & $76.92 \%$ & $65.38 \%$ & $38.46 \%$ & $42.31 \%$ & $61.54 \%$ \\
\hline \% Fixed Financial Performance & $0 \%$ & $0 \%$ & $3.85 \%$ & $0 \%$ & $0 \%$ \\
\hline$\%$ TOTAL & $100 \%$ & $100 \%$ & $100 \%$ & $100 \%$ & $100 \%$ \\
\hline
\end{tabular}

Judging from the BOPO ratio, the highest number of banks that experienced an increase in BOPO (performance decreased) occurred in 2016 and 2017. In that year, as many as 15 banks or $57.69 \%$ of banks experienced an increase in BOPO ratio. In 2018, there were 10 banks or $38.46 \%$ of banks that experienced an increase in the BOPO ratio (decreased performance). 2013 was the year that showed the best performance, with only 6 companies or $23.08 \%$ of companies experiencing an increase in BOPO. 


\section{BELANJA OPERASIONAL TERHADAP PENDAPATAN OPERASIONAL (BOPO)}

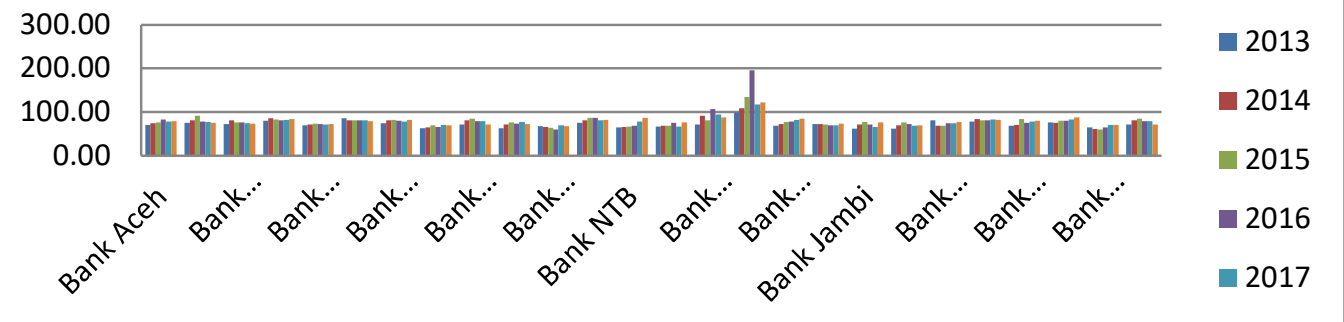

Figure 3. Operational Expenditure On Operating Income (BOPO)

From the bar chart above, it can be seen that fluctuations in the BOPO ratio tend to be stable. Only Bank Banten in 2016 experienced high fluctuations, and the bank also showed the highest average BOPO ratio.

4. Results of Profitability and Profitability Analysis

Table 4. Return on Assets (ROA)

\begin{tabular}{|c|c|c|c|c|c|}
\hline \multirow{2}{*}{ BANK } & \multicolumn{5}{|c|}{ ROA ANALYSIS } \\
\hline & 2014 & 2015 & 2016 & 2017 & 2018 \\
\hline Bank Aceh & Decrease & Decrease & Decrease & Increase & Decrease \\
\hline Bank DKI Jakarta & Decrease & Decrease & Increase & Decrease & Increase \\
\hline Bank Jawa Tengah & Decrease & Decrease & Permanent & Increase & Decrease \\
\hline Bank Jawa Barat & Decrease & Increase & Increase & Decrease & Decrease \\
\hline Bank Kalimantan Barat & Decrease & Decrease & Decrease & Increase & Decrease \\
\hline Bank Sumsel Babel & Increase & Increase & Increase & Decrease & Increase \\
\hline Bank Sumut & Decrease & Decrease & Increase & Decrease & Decrease \\
\hline Bank Bali & Decrease & Decrease & Increase & Decrease & Increase \\
\hline Bank Kaltimtara & Decrease & Decrease & Increase & Decrease & Decrease \\
\hline Bank Sulawesi Tenggara & Decrease & Decrease & Increase & Increase & Increase \\
\hline Bank Sulselbar & Increase & Increase & Increase & Decrease & Increase \\
\hline Bank Sulutgo & Decrease & Decrease & Increase & Increase & Decrease \\
\hline Bank NTB & Decrease & Decrease & Decrease & Decrease & Decrease \\
\hline Bank NTT & Decrease & Decrease & Decrease & Increase & Decrease \\
\hline Bank Papua & Decrease & Increase & Decrease & Increase & Increase \\
\hline Bank Banten & Increase & Increase & Increase & Decrease & Increase \\
\hline Bank Bengkulu & Decrease & Decrease & Decrease & Decrease & Decrease \\
\hline Bank DIY & Increase & Increase & Increase & Decrease & Decrease \\
\hline Bank Jambi & Decrease & Decrease & Increase & Increase & Decrease \\
\hline Bank Jawa Timur & Decrease & Decrease & Increase & Increase & Decrease \\
\hline Bank Lampung & Increase & Decrease & Decrease & Decrease & Decrease \\
\hline Bank Nagari Sumbar & Decrease & Increase & Decrease & Decrease & Increase \\
\hline Bank Riau Kepri & Increase & Decrease & Increase & Decrease & Decrease \\
\hline Bank Kalimantan Selatan & Increase & Decrease & Increase & Decrease & Decrease \\
\hline
\end{tabular}




\begin{tabular}{|c|c|c|c|c|c|}
\hline Bank Kalimantan Tengah & Increase & Increase & Decrease & Decrease & Increase \\
\hline $\begin{array}{l}\text { Bank Kalimantan Timur dan } \\
\text { Utara }\end{array}$ & Decrease & Decrease & Increase & Decrease & Decrease \\
\hline Improved Financial Performance & 8 & 8 & 16 & 9 & 9 \\
\hline Declining Financial Performance & 18 & 18 & 9 & 17 & 17 \\
\hline Fixed Financial Performance & 0 & 0 & 1 & 0 & 0 \\
\hline TOTAL & 26 & 26 & 26 & 26 & 26 \\
\hline $\begin{array}{l}\text { \% Improved Financial } \\
\text { Performance }\end{array}$ & $30.77 \%$ & $30.77 \%$ & $61.54 \%$ & $34.62 \%$ & $34.62 \%$ \\
\hline $\begin{array}{l}\text { \% Declining Financial } \\
\text { Performance }\end{array}$ & $69.23 \%$ & $69.23 \%$ & $34.62 \%$ & $65.38 \%$ & $65.38 \%$ \\
\hline$\%$ Fixed Financial Performance & $0 \%$ & $0 \%$ & $3.85 \%$ & $0 \%$ & $0 \%$ \\
\hline$\%$ TOTAL & $100 \%$ & $100 \%$ & $100 \%$ & $100 \%$ & $100 \%$ \\
\hline
\end{tabular}

The table above shows that 2014 and 2015 were the years with the most decreases in ROA, namely 18 companies, followed in 2017 and 2018. The healthiest conditions were in 2016, where only 9 companies or 34.62\% experienced a decline in performance.

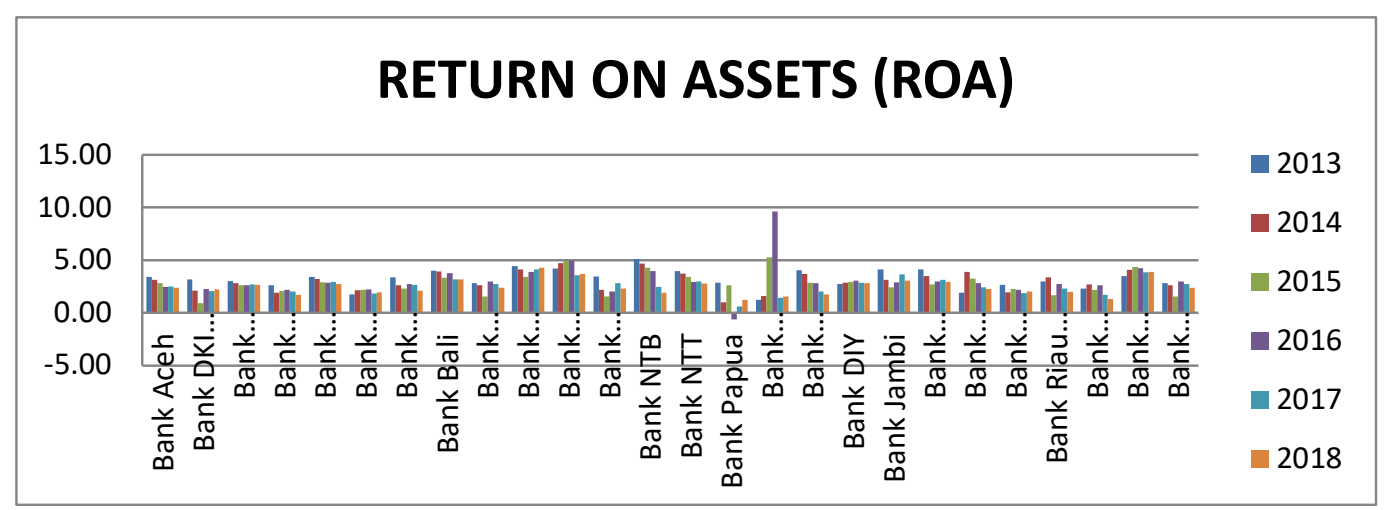

Figure 4. Return on Assets (ROA)

From the diagram above it can be seen that Bank Banten in 2016 experienced a fairly high surge and far above the ROA of other banks, but in subsequent years directly experienced a very significant decline, even ROA of Bank Banten in 2017 and 2018 became more small compared to ROA of other banks. From all banks, it appears that Bank Papua has the smallest ROA compared to other banks.

\section{Liquidity Analysis Results}

Table 5. Loans to Deposit Ratio (LDR)

\begin{tabular}{|l|l|l|l|l|l|}
\hline \multirow{2}{*}{ BANK } & \multicolumn{5}{c|}{ LDR ANALYSIS } \\
\cline { 2 - 6 } & \multicolumn{1}{|c|}{2014} & \multicolumn{1}{c|}{2015} & \multicolumn{1}{c|}{2016} & \multicolumn{1}{c|}{2017} & \multicolumn{1}{c|}{2018} \\
\hline Bank Aceh & Decrease & Increase & Decrease & Increase & Decrease \\
\hline Bank DKI Jakarta & Increase & Increase & Increase & Increase & Decrease \\
\hline Bank Jawa Tengah & Decrease & Decrease & Decrease & Decrease & Decrease \\
\hline Bank Jawa Barat & Increase & Increase & Increase & Decrease & Decrease \\
\hline Bank Kalimantan Barat & Increase & Increase & Decrease & Increase & Decrease \\
\hline Bank Sumsel Babel & Increase & Decrease & Increase & Increase & Increase \\
\hline Bank Sumut & Increase & Increase & Increase & Increase & Decrease \\
\hline Bank Bali & Decrease & Decrease & Decrease & Increase & Increase \\
\hline Bank Kaltimtara & Increase & Decrease & Increase & Increase & Increase \\
\hline Bank Sulawesi Tenggara & Decrease & Increase & Decrease & Decrease & Increase \\
\hline Bank Sulselbar & Increase & Decrease & Increase & Decrease & Decrease \\
\hline
\end{tabular}




\begin{tabular}{|c|c|c|c|c|c|}
\hline Bank Sulutgo & Increase & Decrease & Decrease & Increase & Decrease \\
\hline Bank NTB & Increase & Decrease & Increase & Increase & Decrease \\
\hline Bank NTT & Increase & Decrease & Decrease & Decrease & Decrease \\
\hline Bank Papua & Increase & Decrease & Decrease & Increase & Increase \\
\hline Bank Banten & Increase & Increase & Decrease & Decrease & Increase \\
\hline Bank Bengkulu & Increase & Decrease & Decrease & Increase & Decrease \\
\hline Bank DIY & Decrease & Decrease & Increase & Decrease & Decrease \\
\hline Bank Jambi & Increase & Decrease & Increase & Increase & Incrase \\
\hline Bank Jawa Timur & Decrease & Increase & Decrease & Increase & Increase \\
\hline Bank Lampung & Decrease & Increase & Decrease & Increase & Decrease \\
\hline Bank Nagari Sumbar & Increase & Decrease & Increase & Decrease & Increase \\
\hline Bank Riau Kepri & Increase & Decrease & Decrease & Increase & Decrease \\
\hline Bank Kalimantan Selatan & Decrease & Decrease & Decrease & Increase & Increase \\
\hline Bank Kalimantan Tengah & Decrease & Decrease & Increase & Increase & Increase \\
\hline $\begin{array}{llll}\text { Bank } & \text { Kalimantan Timur dan } \\
\text { Utara } & & & \\
\end{array}$ & Increase & Decrease & Increase & Increase & Increase \\
\hline $\begin{array}{l}\text { Improved Financial } \\
\text { Performance }\end{array}$ & 17 & 9 & 12 & 18 & 12 \\
\hline $\begin{array}{l}\text { Declining Financial } \\
\text { Performance }\end{array}$ & 9 & 17 & 14 & 8 & 14 \\
\hline Kinerja Keuangan Tetap & 0 & 0 & 0 & 0 & 0 \\
\hline TOTAL & 26 & 26 & 26 & 26 & 26 \\
\hline 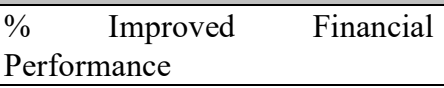 & $65.38 \%$ & $34.62 \%$ & $46.15 \%$ & $69.23 \%$ & $46.15 \%$ \\
\hline $\begin{array}{l}\% \quad \text { Declining } \\
\text { Performance }\end{array}$ & $34.62 \%$ & $65.38 \%$ & $53.85 \%$ & $30.77 \%$ & $53.85 \%$ \\
\hline$\%$ Fixed Financial Performance & $0 \%$ & $0 \%$ & $0 \%$ & $0 \%$ & $0 \%$ \\
\hline$\%$ TOTAL & $100 \%$ & $100 \%$ & $100 \%$ & $100 \%$ & $100 \%$ \\
\hline
\end{tabular}

From the table above it can be seen that the bank's performance in terms of the LDR ratio showed the most decline in performance in 2017, where the number of banks that experienced an increase in LDR was 18 banks or $69.23 \%$ of the total number of banks. Followed in 2014, there were 17 banks or $65.38 \%$ of the total banks. The year 2015 saw the lowest decline in performance, with only 9 banks or $34.62 \%$ experiencing an increase in LDR.

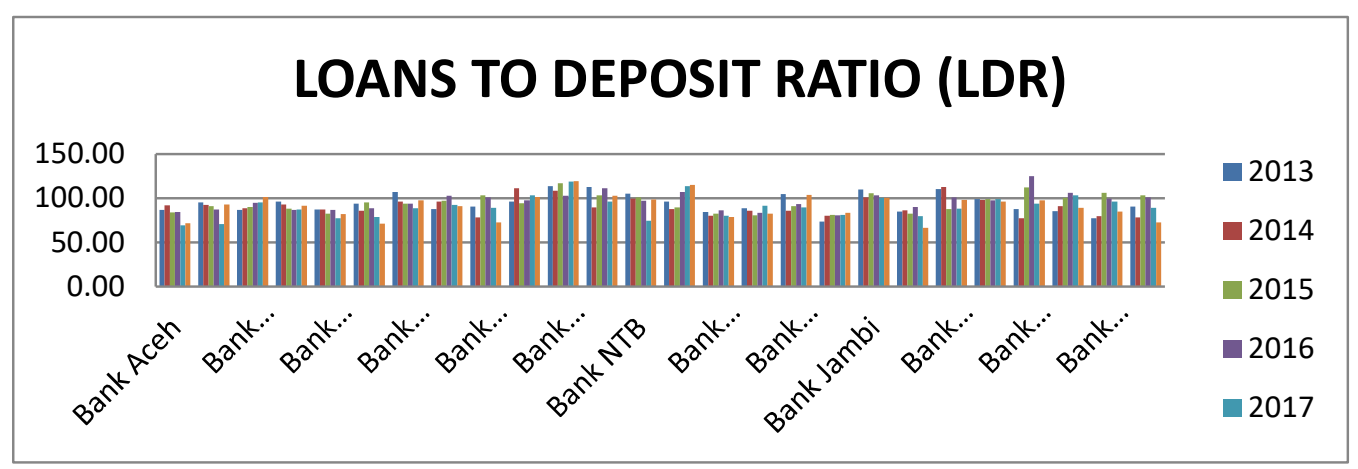

Figure 5. Loans to Deposit Ratio (LDR)

From the bar chart above it can be seen that during the observation period, there were no significant fluctuations. 


\section{Discussion}

\section{Capital Adequacy Ratio}

CAR analysis is a ratio that shows the extent to which a bank's ability to anticipate the need for the availability of its own funds for business growth and bear the risk of losses arising in running its business. The point is CAR is the capital adequacy ratio of a bank in carrying out its business activities. Usually the bank's capital adequacy ratio is compared to the level of credit risk, market risk, and business operational risk. CAR is one of the important indicators measuring the level of quality of bank capital.

\section{Non Performing Loans (NPL)}

NPL is a ratio that shows the level of problem loans compared to the total loans issued. In contrast to CAR, the NPL ratio shows that the biggest increase (decrease in performance) occurred in 2016 and 2018, as many as 15 banks or $57.69 \%$ experienced an increase in NPL (decreased performance). The best NPL performance actually occurred in 2014, with a decrease in NPL occurred in 19 companies or $73.08 \%$. The highest decline in performance in terms of NPLs is still inherent in 2018, still due to the issue of the benchmark interest rate, where an increase in interest rates and weakening the economy will increase the risk of bad credit.

\section{$B O P O$}

The BOPO ratio can provide an assessment of the efficiency of banks, including conventional commercial banks and rural banks. If the bank's BOPO ratio in a certain year has decreased from the previous year, the bank's operations will be more efficient. Conversely, if the bank's BOPO ratio in one year has increased from the previous year, the bank's operations will be increasingly inefficient (BPS, 2017)

Judging from the BOPO ratio, the highest number of banks that experienced an increase in BOPO (performance decreased) occurred in 2016 and 2017. In that year, as many as 15 banks or $57.69 \%$ of banks experienced an increase in BOPO ratio. In 2018, there were 10 banks or $38.46 \%$ of banks that experienced an increase in the BOPO ratio (decreased performance). 2014 was the year that showed the best performance, with only 6 companies or $23.08 \%$ of companies experiencing an increase in BOPO.

If CAR and NPL experience the worst performance decline in 2018, BOPO experienced the worst performance decline in 2016 and 2017. This is because BOPO is a ratio that shows efficiency in managing operational funds, where macro factors are not too influential. Whereas NPL and CAR are ratios that show performance which is strongly influenced by macro factors such as regulation and economic conditions.

\section{$R O A$}

ROA is used to measure the company's ability to generate profits with the total assets (wealth) the company has after adjusting for costs incurred to fund these assets. The higher the ROA, the greater the rate of return on liquid or productive assets4, so the possibility of banks in problematic conditions is smaller. Profitability / profitability analysis is one method of analyzing bank financial performance to measure the ability of banks to generate profits compared to the capital or assets used by the bank concerned to generate profits. In other words, profitability is the ability of a bank to generate profits for a certain period. Analysis of profitability or profitability of a bank illustrates the extent of the success of banks using funds or capital invested to increase profits. To maintain a reasonable or good profitability / profitability level, banks must obtain income that can cover all costs. In addition, the bank must strive to maintain income at a minimum level by taking into account various risks faced (Artarina and Masdjojo, 2013).

\section{Loan to Deposit Ratio}

Liquidity is translated by the LDR indicator (Loan to Deposit Ratio). LDR is a ratio of "credit given to third party funds, which is intended to measure the ability of banks to meet repayments of deposits that are past due to their depositors and can fulfill loan applications submitted without delay" (Kashmir, 2016)

From the calculation results, it can be seen that the bank's performance in terms of LDR ratio showed the most decline in performance in 2017, where the number of banks that experienced an increase in LDR was 18 banks or $69.23 \%$ of the total number of banks. Followed in 2014 , there were 17 banks or $65.38 \%$ of the total banks. The 
year 2015 saw the lowest decline in performance, with only 9 banks or $34.62 \%$ experiencing an increase in LDR.

\section{References}

Artarina, Octa dan Gregorius N. Masdjojo. 2013. Faktor-Faktor Yang Mempengaruhi Rentabilitas Pada Bpr Di Kabupaten Blora. Dinamika Akuntansi, Keuangan dan Perbankan (ISSN : 1979-4878), Mei 2013 Vol. 2, No. 1

Fahmi, Irham. 2011. Analisis Kinerja Keuangan. Bandung: Alfabeta CV

Firdausi, Iqbal. 2016. Analisis Pengaruh Kinerja Perbankan TerhadapDana Pihak Ketiga Bank Persero. Jurnal Keuangan dan Perbankan, Vol.20, No. 3,September 2016. Hal. 487-495. Penerbit STIE Perbanas, Jakarta

Handayani, Puspita Sari. 2005. Analisis Perbandingan Kinerja Bank Nasional, Bank Campuran dan Bank Asing Dengan Menggunakan Rasio Keuangan. Tesis Universitas Diponegoro. Semarang. Hanafi, Mamduh dan Abdul Halim. 2009. Analisis Laporan

Hasan, Amir, Khaerul Anuar, dan Ghafar Ismail. 2010. Studi Pengaruh Makro Ekonomi, Capital, dan Liquidity Terhadap Financial Performance Pada Bank Pembangunan Daerah Di Indonesia Sebelum dan Setelah Otonomi Daerah. Universitas Riau, University Kebangsaan.

https://www.kemenkeu.go.id/publikasi/berita/bpd-diharapkan-jadi-mitra pemerintah-untuk-percepatanpembangunan-daerah/10/08/2018 16:21:19BPD diharapkan Jadi Mitra Pemerintah untuk Percepatan Pembangunan Daerah

Irawan, Bambang BRM. 2011. Analisis Kinerja Bank-bank Pembangunan Daerah yang Ada di Indonesia serta Variabel-variabel yang Mempengaruhinya. Jurnal Ekonomi dan Pembangunan.

Kasmir. 2016. Analisis Laporan Keuangan. Jakarta : PT RajaGrafindo Persad

Peraturan Pemerintah Nomor 71Tahun 2010 tentang Standar Akuntasi Pemerintah

Ponttie, Prasnanugraha. 2007. Analisis Pengaruh Rasio-Rasio Keuangan Terhadap Kinerja Bank Umum di Indonesia. Tesis Universitas Diponegoro. Semarang.

Purwanto, Yahya. 2015. Analisis Perbandingan Kinerja Bank Pembangunan Daerah (BPD) Wilayah Kalimantan dengan Menggunakan Metode CAMEL.

Saragih, Juli Panglima. 2017. Laporan Kinerja Keuangan Bank Pembangunan Daerah dalam Konteks Transparansi dan Akuntabilitas Keuangan Publik. Jurnal BPPK Vol. 10 No.2, Hal. 59-70

Wowiling, F. E. Dan Lisbeth Mananeke. 2018. Analisis Pengaruh Kinerja Keuangan Bank Pembangunan Daerah (BPD) dan Bank Perkreditan Rakyat (BPR) terhadap Pemberian Kredit Periode 2013-2016. Jurnal $E M B A$. Vol.6 No.1 Januari 2018, Hal.81-90 